\title{
Effects of Canine and Murine Mesenchymal Stromal Cell Transplantation on Peripheral Nerve Regeneration
}

\author{
Diego Noe Rodriguez Sanchez, Matheus Bertanha ${ }^{2}$, Thiago Dias Fernandes ${ }^{3}$, \\ Luiz Antônio de Lima Resende ${ }^{3}$, Elenice Deffune ${ }^{4}$, Rogério Martins Amorim ${ }^{1}$ \\ ${ }^{I}$ Department of Veterinary Clinics, School of Veterinary Medicine and Animal Science, São Paulo State University (UNESP), \\ ${ }^{2}$ Department of Surgery and Orthopedics, Vascular Laboratory, ${ }^{3}$ Department of Neurology and Psychiatry, ${ }^{4}$ Blood Transfusion Center, \\ Cell Engineering Laboratory, Botucatu Medical School, São Paulo State University (UNESP), Botucatu, SP, Brazil
}

Background and Objectives: Maintaining a permissive microenvironment is essential for adequate nerve regeneration. Cell-based therapy has the potential based cell replacement and promotion of axonal growth. The adipose tissue derived mesenchymal stromal cells (Ad-MSC) attract interest because neuroregenerative and anti-inflammatory properties. The aim of this study was to evaluate the effects of canine and murine Ad-MSC transplantation on the sciatic nerve regeneration.

Methods: Forty Wistar rats were divided randomly into: control group - CG $(n=8)$; denervated group - DG $(n=8)$; decellularized vein group - VG $(n=8)$; decellularized vein + canine MSC-cMSC $(n=8)$; descellularized vein + murine MSC-mMSC ( $\mathrm{n}=8)$. After 10-mm nerve gap, the tubulation technique was performed with decellularized vein filled with $10^{6}$ MSC labeled with quantum dots $\left(\mathrm{Qtracker} 665^{\mathbb{R}}\right.$ ). The sciatic nerve functional index (SFI) and electroneuromyography (ENMG) measurements were carried and morphometric and immunohistochemistry analysis of the tissue. Results: The SFI values were higher in the cMSC and mMSC groups at day $27(p<0.020)$ and day $35(p<0.011)$. The ENMG analysis also revealed better results in the mMSC group. Density, number, and total area of the fibers were increased in the mMSC and cMSC groups. Brain-derived neurotrophic factor BDNF and S-100 protein positive immunoreactivity showed a higher expression for both in the nerve of the $\mathrm{mMSC}$ and cMSC groups. The MSC labeled with quantum dots were detected at day 35, indicating neuronal survival long after the nerve damage.

Conclusions: Murine and canine Ad-MSC associated with decellularized vein scaffold had positive effects on sciatic nerve regeneration in rats.

Keywords: Regenerative medicine, Cell-based therapy, Nerve regeneration, Sciatic nerve

Accepted for publication October 20, 2016, Published online May 30, 2017 Correspondence to Rogério Martins Amorim

Department of Veterinary Clinics, College of Veterinary Medicine and Animal Science, São Paulo State University (UNESP), Prof Doutor Walter Mauricio Correia Street, UNESP Campus de Botucatu, 18618-681-Botucatu, SP, Brazil

Tel: +55-14-38802082, Fax: +55-1438152343

E-mail: rmamorim@fmvz.unesp.br

(a) This is an open-access article distributed under the terms of the Creative Commons Attribution Non-Commercial License (http://creativecommons.org/ licenses/by-nc/4.0/), which permits unrestricted non-commercial use, distribution, and reproduction in any medium, provided the original work is properly cited.

Copyright (C) 2017 by the Korean Society for Stem Cells Research

\section{Introduction}

After complete nerve transection, a process of axonal degeneration characterized by myelin fragmentation and separation occurs (1), which typically results in poor and incomplete recovery of functionality. Although the peripheral nervous system is capable of regeneration after injury, severe lesions prevent the direct reconnection of nerve stumps. Nerve autograft is considered the gold standard technique in treating nerve injuries, but it presents a few disadvantages including injury to the donor nerve and issues with re-innervation and poor vascularization. Main- 
taining a permissive environment in the nerve after injury is crucial to stimulate neuroregeneration $(2,3)$. In order to repair nerve injury, tubulation techniques aim to guide fibers sprouting, supply extracellular matrix molecules, neurotrophic factors and cells, thus creating a microenvironment for regeneration $(3,4)$. There are many tubulation techniques, which use several different tissue-engineered scaffolds, such as collagen, polycaprolactone, polyglycolic acid, silicone, laminin, and arteries, veins, and muscle fibers (5-9).

Cell-based therapies, especially the ones utilizing mesenchymal stromal cells (MSC), have become the focus of attention for the treatment of nerve injury, owing to their positive effects in the regeneration process $(7,8,10-14)$.

Several studies have shown that the MSC transplantation in nerve injury promotes the replacement and fusion with the damaged Schwann cells, leading to remyelination. Moreover, it provides paracrine effects amplifying the production of neurotrophic factors and contributing to axonal protection; it also releases growth factors, has immunomodulatory and anti-inflammatory effects, provides physical support for axonal growth, and aids energy production for axonal metabolism $(6,10,11,15)$.

In this context, the purpose of this study was to investigate and compare the neuroregenerative effects of combined use of decellularized vein containing murine and canine adipose-derived mesenchymal stromal cells (Ad-MSC) on a $10-\mathrm{mm}$ sciatic nerve gap in a rat model.

Our hypothesis is that the combined use of decellularized vein with Ad-MSC in transected nerves stimulates axonal regeneration, improving the functional, electrophysiological, and morphological aspects.

\section{Materials and Methods}

\section{Experimental animals}

Forty Wistar rats were used in this study, 20 males and 20 females, weighing between 200 and $300 \mathrm{~g}$, with a mean age of 90 days. They were maintained under controlled temperature, humidity and light conditions, receiving water and food "ad libitum". All procedures were approved by the ethics committee for animal research (CEUA), from Botucatu Medical School/UNESP, Brazil (protocol FMB-PE-2/2015).

\section{Experimental design}

In order to observe the effects of the tubulation technique with both canine and murine MSC after neurotmesis, we randomly allocated the rats into five groups. Group 1 normal control group (CG) $(n=8)$; received only the nerve sciatic posterior approach. Group 2 - denervation control (DG) $(n=8)$; received the sciatic nerve posterior approach and the nerve was denervated. Group 3 - decellularized vein $(\mathrm{GV})(\mathrm{n}=8)$; surgical procedure was performed to prepare for tubulation of the injured nerve with a conduit whose lumen was filled with HBSS solution. Group 4 vein + canine mesenchymal stromal cells transplantation (cMSC) $(\mathrm{n}=8)$; surgical procedure was performed to prepare for tubulation of the injured nerve with a conduit whose lumen was filled with suspension containing cMSC. Group 5 - Vein + murine mesenchymal stromal cells transplantation (mMSC) $(n=8)$; surgical procedure was performed to prepare for tubulation of the injured nerve with a conduit whose lumen was filled with suspension containing mMSC.

The sciatic nerve functional index (SFI) was measured before surgery (pre-op), and after surgery at day 9, 18, 27 and 35. Additionally, the sciatic nerve functionality was evaluated by electroneuromyography (ENMG) at the pre-surgery and at day 35 post-surgery. Rats were euthanized at day 35 post-surgery, and the sciatic nerves were isolated in order to perform immunohistochemistry and morphometric studies. The weight of the tibial cranial (TCM) and gastrocnemius muscles of both limbs were also measured.

\section{Tubulation technique and MSC transplantation}

The rats from the DG, VG, cMSC and mMSC groups received inhalation anesthesia with isoflurane, and a posterior approach to the left sciatic nerve was performed in order to induce neurotmesis (Sunderland 5), removing 10 $\mathrm{mm}$ of nerve length under surgical microscope $(10,16)$. The right sciatic nerves of all the rats were left untouched to be used as control for the immunohistochemistry analysis. The sciatic nerves of the DG group were sectioned, directed $90^{\circ}$ and sutured to the adjacent muscle to avoid reinnervation. The VG, cMSC and mMSC groups underwent the tubulation technique using the decellularized vein scaffold, previously prepared according to established protocols (17). The vein was fixed with perineural suture, using the polypropylene 7/0 (Ethicon, Somerville, USA), between the proximal and distal stumps, inserting the stumps $2 \mathrm{~mm}$ into the vein.

The vein lumens of the VG group were filled with 13 $\mu \mathrm{L}$ of HBSS buffer (Hank's balanced salt solution, Gibco ${ }^{\circledR}$ HBSS, Thermo Fisher Scientific, São Paulo, Brazil) using a Hamilton microsyringe. The lumens of the veins for the mMSC and cMSC groups were filled with $13 \mu \mathrm{L}$ of solution containing $10^{6}$ cells labeled with the nanocrystal Qtracker ${ }^{R} 655$ (Life Technologies Corporation, Carlsbad, USA), according to the manufacturer's protocol, and mi- 
croencapsulated into the platelet gel activated with calcium gluconate $(40 \mathrm{mM}, 1 \mu \mathrm{L})$ and thrombin $(50 \mathrm{U} / \mathrm{cc}$, $2 \mu \mathrm{L})$. For gel platelet preparation, samples of whole blood were collected in tubes with citrate phosphate dextrose anticoagulant in 1:10 proportion, according to established protocols (18).

After surgery, the animals were treated with non-steroidal anti-inflammatories, opioid analgesics, and antibiotics.

\section{Isolation and characterization of the Ad-MSC}

Canine and murine Ad-MSC were isolated from subcutaneous adipose tissue, dissociated enzymatically with collagenase type 1 (0.04\%) (Sigma-Aldrich, Saint Louis, USA), and separated by centrifugation and filtration. The cells were re-suspended in 90\% Dulbecco's Modified Eagle Medium (DMEM) high glucose, 10\% fetal bovine serum (FBS), 1\% penicillin/streptomycin (10,000 U/mL), amphotericin B 1.2\% (3 mg/mL) (Invitrogen, São Paulo, Brazil), $0.005 \%$ amikacin $(250 \mathrm{mg} / \mathrm{mL})$ (Novafarma, Anapolis, Brazil) and cultivated in flasks. Cells were trypsinized (trypsin, 0.25\%, Invitrogen, São Paulo, Brazil) when they reached $80 \%$ confluence, according to previously published protocols (17).

The canine and murine MSC were analyzed by the BD FACSCalibur flow cytometer (Becton Dickinson Company, San Jose, USA). The mMCS were evaluated for the expression of the following positive surface markers: CD 90-PERCP anti rat (Becton Dickinson Company ${ }^{\mathrm{TM}}$ ), CD44-FITC anti rat (Becton Dickinson Company ${ }^{\mathrm{TM}}$ ), and negative ones: CD11b-APC (Becton Dickinson Company ${ }^{\mathrm{TM}}$ ), CD31-PE anti rat (Becton Dickinson Company ${ }^{\mathrm{TM}}$ ), and CD45-PE anti rat (Pharmingen). The cMSC were evaluated for the expression of the following positive surface markers: CD44 rat anti-mouse (Sigma-Aldrich), CD90-FITC mouse anti-human (Becton Dickinson Company ${ }^{\mathrm{TM}}$ ), and CD105 mouse anti-human (Abcam ${ }^{\mathrm{TM}}$ ), and of the following negatives: CD34-FITC mouse anti-human (Becton Dickinson Company ${ }^{\mathrm{TM}}$ ), and CD45 rat anti-dog (abd Serotec $\left.^{\mathrm{TM}}\right)$. Aliquots of cells were incubated with antibodies following the manufacturer protocols. The canine and murine samples contained $2 \times 10^{5}$ and $1 \times 10^{5}$ cells $/ \mathrm{mL}$, respectively. Data were evaluated in the Cell Quest Pro software (Becton Dickinson Company, San Jose, USA).

The canine and murine Ad-MSC showed plastic adherence, fibroblast morphology, differentiation for the adipogenic, chondrogenic and osteogenic lineages, and surface marker expression by flow cytometer for CD34-, CD45-, $\mathrm{CD} 44+, \mathrm{CD} 90+$, and CD105 - for canine Ad-MSC (Fig. 1), and $\mathrm{CD} 11 \mathrm{~b}-, \mathrm{CD} 31-, \mathrm{CD} 45-, \mathrm{CD} 44+$, and $\mathrm{CD} 90+$ for murine Ad-MSC.

\section{Cell viability}

The transplanted murine and canine Ad-MSC had 70 $80 \%$ cell viability, as assessed by $0.3 \%$ trypan blue exclusion test, immediately prior to transplantation. The result was expressed as a percentage of viable cells according to the formula: Viability $(\%)=$ cells stained (viable) $\times 100 /$ total cells (stained + unstained).

\section{Sciatic Functional Index (SFI)}

The SFI was measured at day 9, 18, 27, and 35 after surgery in the CG, DG, VG, cMSC, and mMSC groups. The hindlimbs of each animal were dipped in Indian black ink and the animals were tested in a confined walkway measuring $78 \times 9 \mathrm{~cm}$, on a white paper where the footprints were taken. Several measurements were obtained from the footprints: (i) distance from the heel to the third toe, the print length (PL); (ii) distance from the first to the fifth toe, the toe spread (TS); and (iii) distance from the second to the fourth toe, the intermediary toe spread (ITS). These parameters were measured for both normal (N) and experimental (E) feet. The SFI were analyzed following the equation: $-38.3([\mathrm{EPL}-\mathrm{NPL}] / \mathrm{NPL})+109.5$ $([\mathrm{ETS}-\mathrm{NTS}] / \mathrm{NTS})+13.3([\mathrm{EIT}-\mathrm{NIT}] / \mathrm{NIT})-8.8$ according to (19). A SFI value of -100 means total transection, while a SFI value of 0 means normal function.

\section{Electroneuromyography (ENMG)}

Nerve signal latency $(\mathrm{m} / \mathrm{s})$ and amplitude $(\mathrm{mV})$ were measured in the CG, DG, VG, cMSC, and mMSC groups
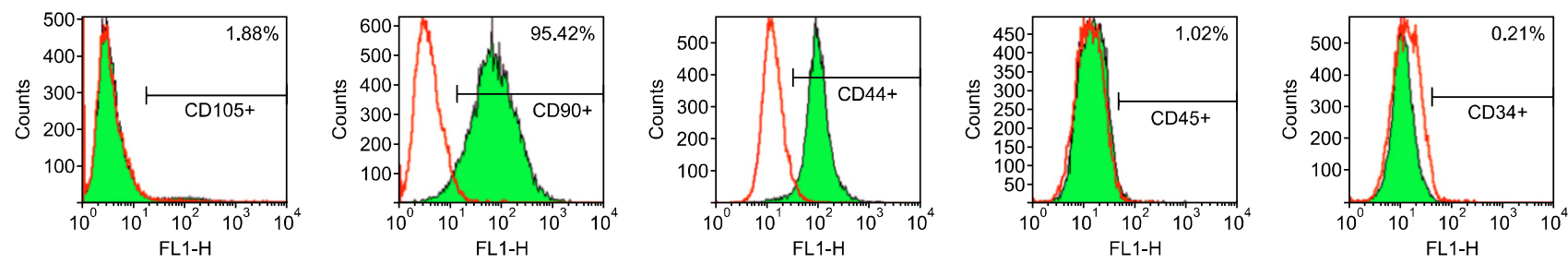

Fig. 1. Surface markers expression in the canine Ad-MSC. The white area indicates the negative control of the respective marker and the green area represents the sample tested. 
immediately before the experimental neurotmeses and at day 35 after the procedure. An active surface electrode (black) and a reference electrode (red) were placed in the center and near the insertion of the left cranial tibial muscle tendon, respectively. Furthermore, a dispersive electrode (green) was located in the ventral abdominal region. A bipolar stimulation electrode was positioned in the proximal stump of the sciatic nerve. A Sapphire II $4 \mathrm{ME}$ instrument (Teca Medelec, USA) was used; the intensity ranges of stimulation and filter were $10 \sim 20 \mathrm{~mA}$ and $20 \sim$ 2,000 Hz.

\section{Morphological study}

Morphological analysis to measure the number and density of nerve fibers and the total nerve diameter, were performed at day 35 after surgery in the CG, DG, VG, cMSC, and mMSC groups. The animals were humanely sacrificed to dissect the sciatic nerve. Additionally, samples were collected of the left (atrophy) and right (normal) hind limb muscles (cranial tibial and gastrocnemius) to compare their weight. Samples of the distal portion of the sciatic nerves were fixed in $10 \%$ buffered formalin for $48 \mathrm{~h}$, included in paraffin, and semi-thin sections $(5 \mu \mathrm{m})$ were cut and stained with osmium tetroxide $1 \%$. The images were obtained using an inverted light microscope (DM 4000 B F; Leica Microsystems, Wetzlar, Germany).

The number of nerve fibers $(\mathrm{N})$, their density $\left(\mathrm{N} / \mathrm{mm}^{2}\right)$, and the total nerve diameter $\left(\mu \mathrm{m}^{2}\right)$ were measured obtaining four fields in each slide with $1000 \times$ magnification for analysis using ImageJ software (National Institute of Health, USA) according to previously described protocols (20).

Fragments of the central portion of the sciatic nerve in the cMSC and mMSC groups were collected for tracking the cells labeled. Samples were included in OCT (Optimal critical temperature), followed by slicing into $10-\mu \mathrm{m}$ thickness sections, in a cryostat (CM-1850 model; Leica Microsystems) and analyzed with a fluorescence microscope (DM LB, Leica Microsystens) with a wavelength of $655 \mathrm{~nm}$ at magnification $10 \times$ and $40 \times$.

\section{Immunohistochemistry (IHC)}

Brain-derived neurotrophic factor (BDNF) and S-100 protein expression in the sciatic nerve were measured at day 35 after surgery in the CG, DG, VG, cMSC, and mMSC groups. HiDef ${ }^{\mathrm{TM}}$ HRP polymer system (Cell Marque $^{(\mathbb{R}}$ ) was used with diaminobenzidine as chromogen, using their positive and negative controls. The antibodies anti-S100 (Sigma-Aldrich; dilution 1:1000) and anti-BDNF (Abcam; 1:500 dilution) were used. The intensity of the immunohistochemical reaction was quantified using the ImageJ software (National Institutes of Health, USA) as previously described (21).

\section{Statistical analysis}

All variables were analyzed independently for the normal distributions. Means (parametric) or medians (non-parametric) were compared among groups and moments. Wilcoxon test for paired and Friedman test for repeated measures were used. Non-repeated and unpaired measures were analyzed with Mann-Whitney and Kruskal Wallis tests, respectively (GraphPad Prism version 5.01 for Windows, San Diego, California, USA). p values of less than 0.05 were considered statistically significant.

\section{Results}

\section{Sciatic Functional Index (SFI)}

No significant differences in the SFI among the experimental groups at day 9, 18, and $27(\mathrm{p}>0.05)$ were found, although the mMSC group showed median values closer to those of the CG. However, at day 35, significant differences were observed between the mMSC (-53.58) group and the DG $(-90.00), \mathrm{VG}(-86.73)$, and cMSC $(-86.60)$ groups $(\mathrm{p}<0.05)($ Fig. 2$)$.

\section{Electroneuromyography (ENMG)}

Latency: The mean latency values for the nerve signal in both DG and VG groups at day 0 were $1.73 \mathrm{~m} / \mathrm{s}$. At

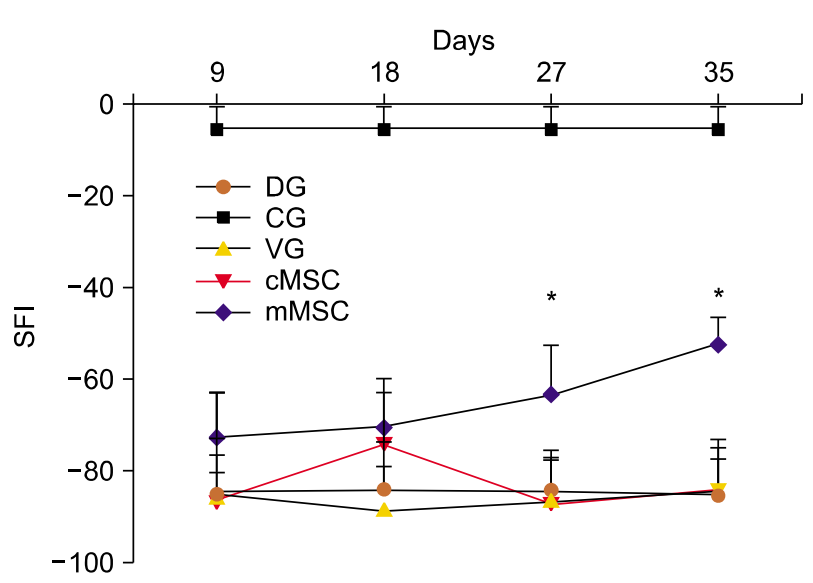

Fig. 2. Graph representing the typical footprint obtained from a normal animal with their respective variables and gait assessment. The effect of MSC transplant on the nerve sciatic function was determined in CG $(n=7)$, DG $(n=7)$, VG $(n=7)$, cMSC $(n=7)$, mMSC $(\mathrm{n}=7)$ on $9,18,27$ and 35 days after surgery ( $1 \sim 4$ weeks). *Difference among the groups $(p<0.05$ tested by repeated measures Friedman). 
day 35 , the latency was not obtained due to a complete absence of electrical conduction of the sciatic nerve, indicating neurotmesis.

The mean latency values for the nerve signal in the cMSC group at days 0 and 35 were $1.75 \mathrm{~m} / \mathrm{s}$ and $3.1 \mathrm{~m} / \mathrm{s}$ $(\mathrm{p}<0.05)$ respectively. The mean latency values for the nerve signal in the cMSC groups at 0 and 35 were 1.61 $\mathrm{m} / \mathrm{s}$ and $1.73 \mathrm{~m} / \mathrm{s}(\mathrm{p}>0.05)$, respectively. These results showed that the mMSC latency remained closer to control values.

Amplitude: The median amplitude values for the nerve signal in the DG and VG groups at days 0 and 35 were $15.03 \mathrm{mV}$ and $0.0 \mathrm{mV}(\mathrm{p}<0.05)$, and $16.95 \mathrm{mV}$ and 0.0 $\mathrm{mV}(\mathrm{p}<0.05)$, respectively. This strongly suggests that there were no myelinated fibers reaching the muscle.

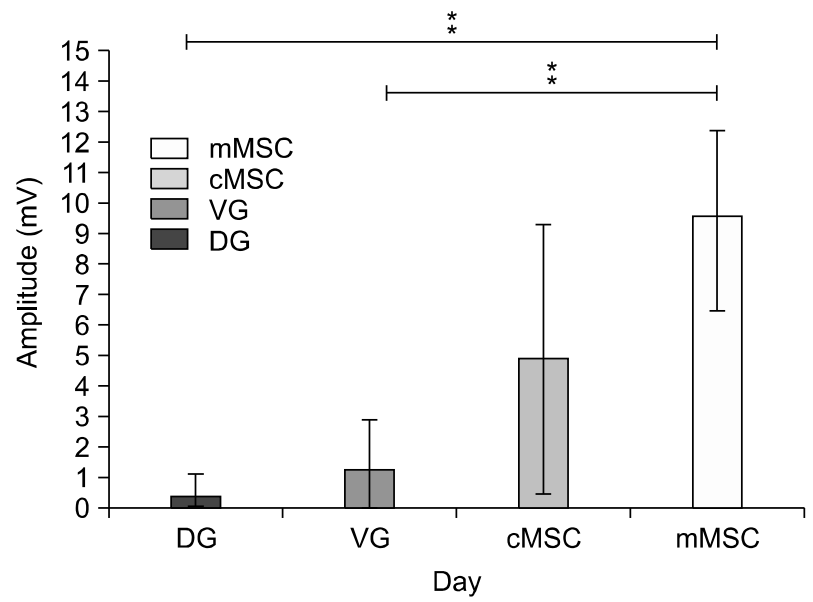

The mean amplitude values for the nerve signal in the cMSC and mMSC groups at days 0 and 35 were $16.95 \mathrm{mV}$ and $3.23 \mathrm{mV}(\mathrm{p}<0.05)$, and $17.45 \mathrm{mV}$ and $9.76 \mathrm{mV}(\mathrm{p}<0.05)$, respectively.

When the mean amplitude values were compared among the experimental groups at day 35 , significant differences were observed among the mMSC $(9.76 \mathrm{mV})$ and DG $(0.00 \mathrm{mV}), \mathrm{VG}(0.00 \mathrm{mV})$, and cMSC (3.23 mV) groups, suggesting that the mMSC group maintained the amplitude closer to the control (pre-op) values (Fig. 3).

Morphological study: The mean values of the density and number of nerve fibers at day 35 were $2.2 \pm 0.71 / \mathrm{mm}^{2}, 431.5$ for DG; $3.65 \pm 2.65 / \mathrm{mm}^{2}, 1,353$ for $\mathrm{VG} ; 4.8 \pm 1.97 / \mathrm{mm}^{2}, 2,802$ for cMSC; $2.43 \pm 0.29 / \mathrm{mm}^{2}, 1,499$ for mMSC, respectively. The difference in number of fibers between the DG and

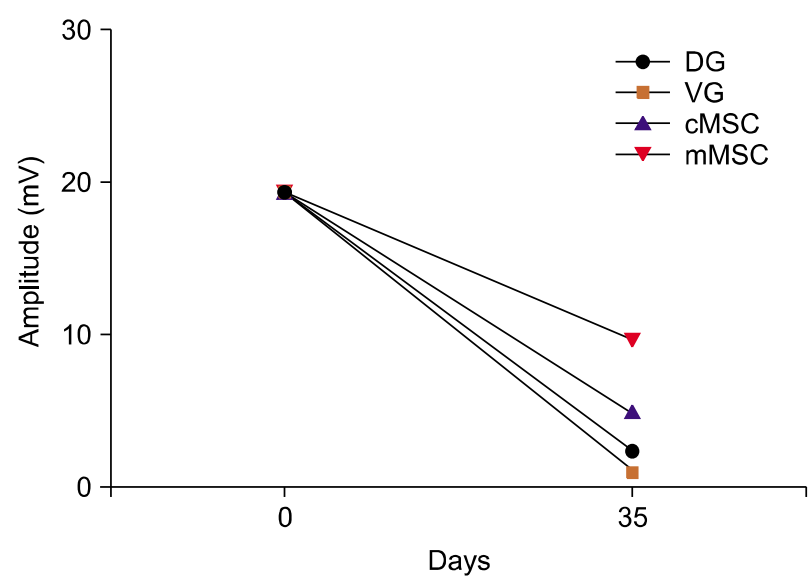

Fig. 3. Graph depicting the distal amplitude of the experimental groups on day 0 before the experimental procedures and day 35 after repair of the left sciatic nerve. The effect of MSC transplantation on the sciatic nerve on ENMG was determined in DG $(n=7)$ CG $(n=7)$, VG $(n=7)$, cMSC $(n=7)$, mMSC $(n=7)$. ${ }^{*}$ Difference among the groups $(p<0.05)$.

A

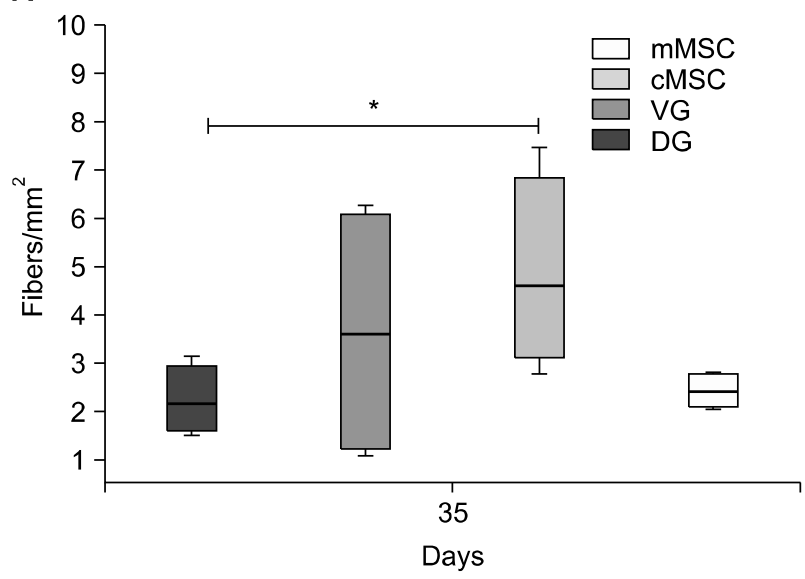

B

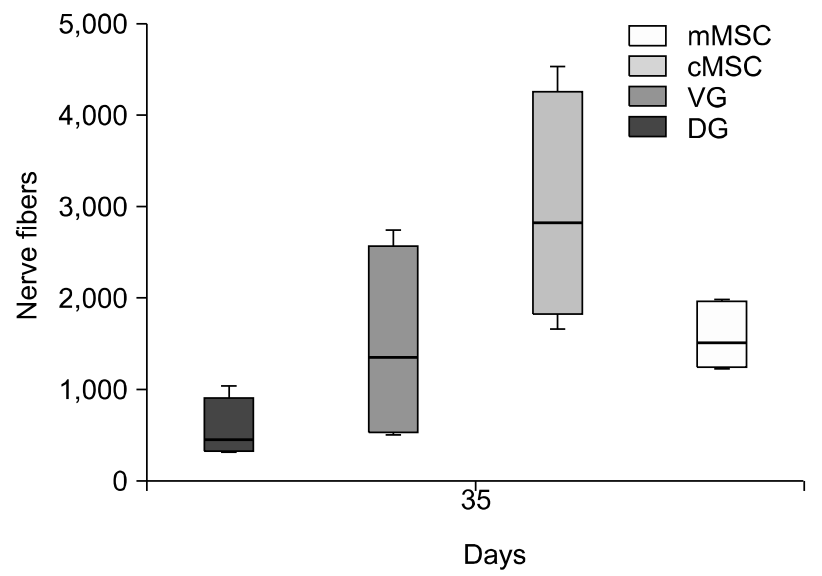

Fig. 4. Graphs representing the density of nerve fibers (A) and the total number of fibers (B). Groups CG $(n=7)$, DG $(n=7)$, VG ( $n=7)$, cMSC $(n=7)$ and mMSC $(n=7)$, at the end time of the experiment (35 days). *Difference among the groups $(p<0.05)$. 

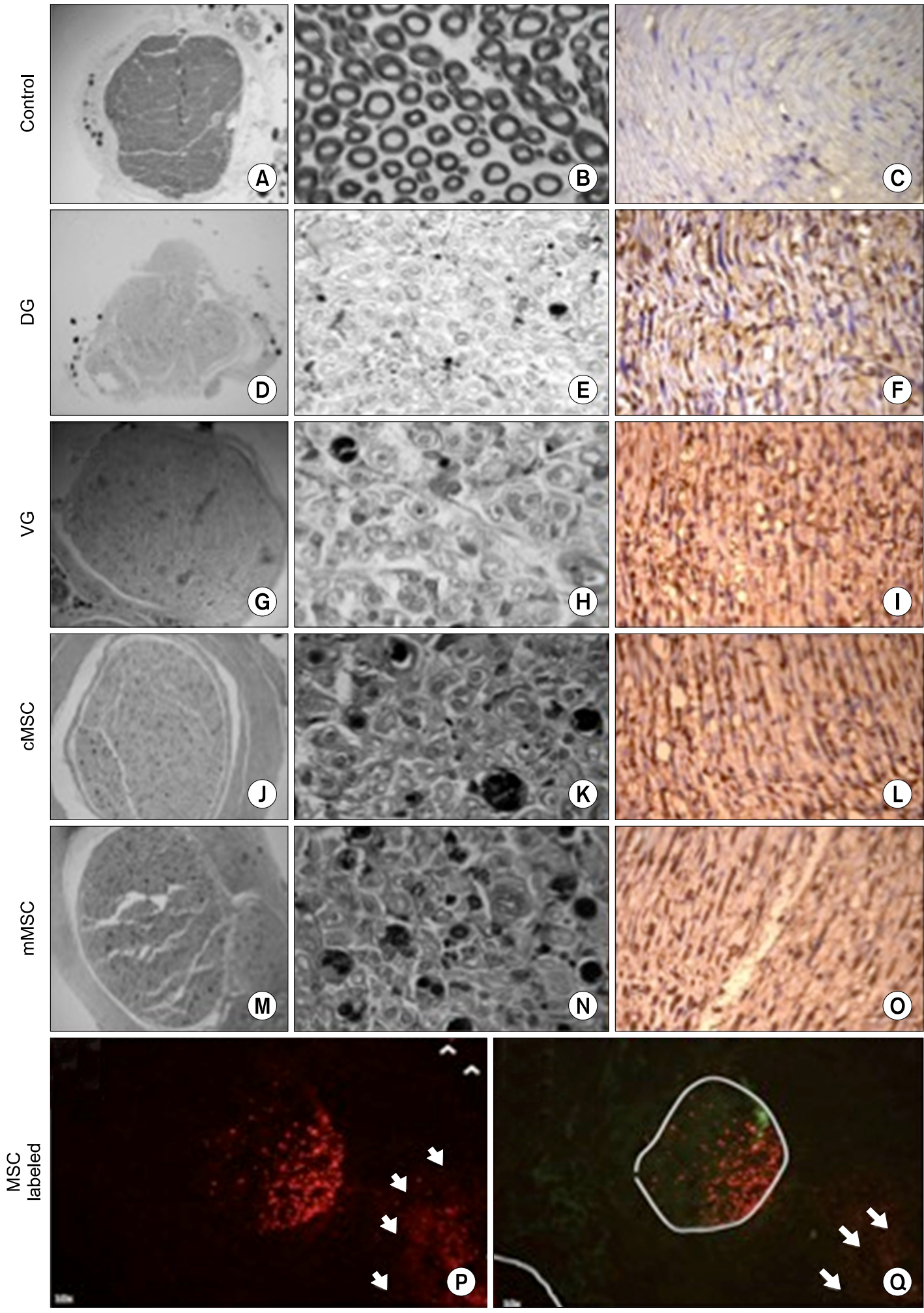

Fig. 5. Panoramic and zoom of the distal portion of the sciatic nerve injured in the CG (A, B, C), DG (D, $E, F), V G(G, H, I) c M S C$ $(J, K, L) \operatorname{mMSC}(M, N, O)$ groups, showing the overall diameter nerve fibers $(A, D, G, J, M$, magnification $10 \times)$, axon density $(B, E$, $\mathrm{H}, \mathrm{K}, \mathrm{N}$, magnification, 100 $\times$ ) and positive S-100 expression (C, F, I, L, O, magnification 40×). The persistence of labeled Ad-MSC was observed in the proximal portion of the sciatic nerve in the CMSC group $(\mathrm{P}, \mathrm{Q}$, magnification $10 \times)$. The arrows represent the cMSC. In the demarcated region is observed CMSC concentration, mainly in the proximal region $(\mathrm{Q})$. Note the cell migration to the distal stump (arrows). cMSC migrating to the proximal region (arrowhead). 
cMSC groups was statistically significant $(\mathrm{p}<0.05)($ Fig. 4).

The mean values of the total area of nerve fibers at day 35 , were $270,322 \pm 184,780 \mu \mathrm{m}^{2}$ for DG; 437,706 $\pm 164,532 \mu \mathrm{m}^{2}$ for VG; $629,692 \pm 105,336 \mu \mathrm{m}^{2}$ for cMSC, $600,477 \pm 53,063 \mu \mathrm{m}^{2}$ for $\mathrm{mMSC}$, respectively. The difference in total area of nerve fibers for the cMSC and mMSC groups compared to the DG group was statistically significant $(\mathrm{p}<0.05)$.

Morphological analysis of the sciatic nerves revealed mononuclear cells infiltrates and axonal degeneration in all experimental groups, characterized by the presence of myelin breakdown and disintegration, digestion chambers, Schwann cells proliferation, compartmentalization, mini fascicles degeneration surrounded by perineurium, and greater invasion with connective tissue, thus losing the architecture of a healthy nerve. However, improvements in some morphological aspects, including axonal sprouting and presence of myelinated fibers, were observed at the distal portion of the sciatic nerve injured in the $V G$, cMSC, mMSC groups, when compared with DG group (Fig. 5).

The persistence of labeled Ad-MSC was observed in the proximal portion of the injured sciatic nerve in the cMSC and mMSC group, indicating the engraftment of the Ad-MSC in the nerves (Fig. 5).

The weights of the left cranial tibial and gastrocnemius muscles at day 35 were significantly lower in all experimental groups $(\mathrm{p}<0.05)$, when compared with the right side (Table 1). However, comparing the groups, there were no statistically significant differences neither in the gastrocnemius $(\mathrm{p}>0.05)$, nor in the tibial cranial muscle $(\mathrm{p}>$ $0.05)$.

Immunohistochemistry: The intensity of the positive BDNF expression at day 35 was $0.14,0.17,0.32$, and 0.29 for $\mathrm{DG}, \mathrm{VG}, \mathrm{cMSC}$, and mMSC groups, respectively. Statistically significant differences were observed between mMSC and DG $(p<0.05)$ (Fig. 6). The expression of S-100 protein was higher in the cMSC and mMSC, when compared to DG and VG (Fig. 5).

\section{Discussion}

Creating an adequate microenvironment in the peripheral nerve after injury is crucial for the success of the neuroregeneration process. In this context, the cellular constituents and the geometric organization of components and neurotrophic factors play an essential role in nerve repair.

Although Schwann cell transplantation has been shown to enhance the nerve regeneration process, there are limitations to this approach related to the difficulties in ob-

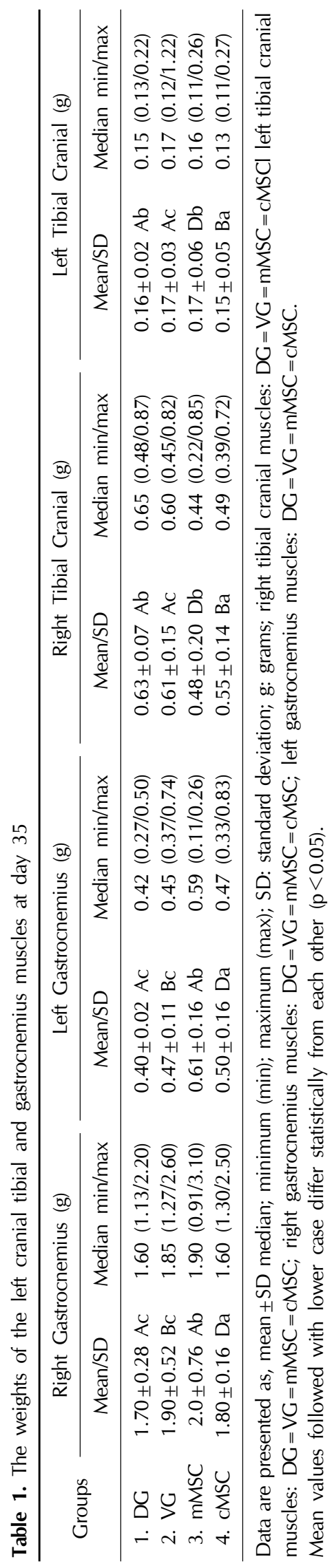




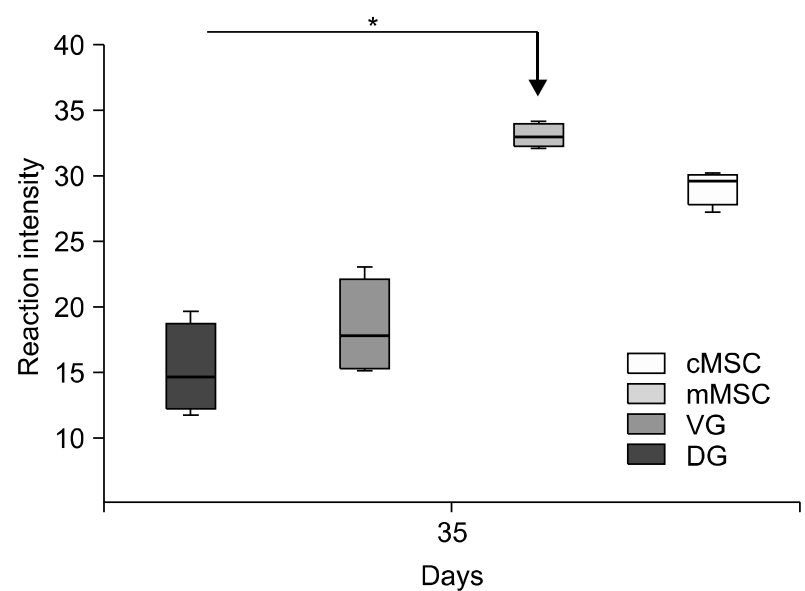

Fig. 6. Graph representing immunohistochemistry staining with BDNF. DG $(n=7)$ control group $(n=7)$, VG $(n=7)$, cMSC $(n=7)$, mMSC $(n=7)$ on day 35 after the repair of the left sciatic nerve. *Difference among the groups $(p<0.05)$.

taining and culturing Schwann cells $(3,22)$. In contrast, the MSCs are multipotent cells, easily obtained and cultured, with self-renewal capacity, high growth rate, and paracrine effects (10-12). Several studies have confirmed the positive effects of MSCs in the peripheral nerve regeneration process $(4,6,10,12,23)$. Ad-MSCs have been used in combination with tubulation techniques, injected directly into the conduit with the expectation that the secretion of paracrine factors, together with cell replacement and the physical support by the conduit, would stimulate nerve regeneration $(3,4,10,23-27)$.

SFI is a gait assessment test that analyzes the motor activity related with pelvic limb muscles innervated by cranial tibial and common peroneal nerves (16, 23, 25, 28). In our study, the mMSC group showed a positive effect on the SFI at day 35, in agreement with the results obtained in the ENMG and in the morphological study.

The groups treated with Ad-MSCs revealed better results in the ENMG amplitude than the VG and DG groups, indicating that more myelinated fibers were regrowing, generating an electrophysiological response. Moreover, the mMSC and cMSC groups maintained a latency value closer to that of the $\mathrm{CG}$, indicating that the treatment may have contributed to the remyelination of damaged nerve. This statement is reinforced by the SFI results and by the increase in the number, area, and density of the nerve fibers of the distal portion of the sciatic nerve in the cMSC and mMSC groups, thus suggesting the presence of myelinated axonal sprouting, as reported elsewhere $(19,26,27,29,30)$. It is likely that these positive effects were associated with the enhancement of the microenvironment, mediated by neurotrophic factors such as
BDNF, secreted by Ad-MSCs. Moreover, the presence of MSCs labeled with nanocrystal Qtracker ${ }^{\mathbb{R}} 655$ in the cMSC and mMSC groups supports these findings.

Neurotrophic factors have been shown to stimulate nerve regeneration in vitro and in vivo, in experimental models of axonotmesis and neurotmesis, thus playing a neuroregenerative and neuroprotective role. The neurotrophin family of proteins (BDNF, Nerve Growth Factor [NGF], Neurotrophin-3,4,5 [NT-3,4,5]) and their receptors $\left(\mathrm{P} 75^{\mathrm{NTR}}\right.$ and Trk [tropomyosin-related kinase] receptor tyrosine kinase) stimulate the assembly of the cytoskeletal components $(31,32)$. According to Hoke et al., 2002 and Fu\&Gordon, 1997 (33, 34), the expression of BDNF and NGF receptors is upregulated a few days after nerve injury, followed by downregulation at approximately 4 weeks. In this context, the highest expression of BDNF observed in our study at day 35 in the cMSC and mMSC groups suggests that the transplanted Ad-MSC secrete these neurotrophins as shown in others studies $(4,30,35)$.

MSC secrete extracellular matrix components, growth factors, and inflammatory regulators found in soluble forms and exocytic vesicles (exosomes) (36). Bone marrow-derived MSC (BM-MSC), adipose tissue-derived MSC (AT-MSC), and umbilical cord-derived MSC (UC-MSC), all have been shown to be capable of secreting to the surrounding medium neuroregulatory factors, such as NGF, BDNF, NT-3, NT-4/5, basic Fibroblast Growth Factor (bFGF), Vascular Endothelial Growth Factor (VEGF), Insulin-like Growth Factor (IGF), and Glial-Derived Neurotrophic Factor (GDNF) (36, 37).

Several studies have reported that MSC under appropriate stimulus and microenvironmental conditions can differentiate into Schwann-like cells (7). Moreover, an in vivo study has demonstrated an increase in the expression of the S-100 protein and $\mathrm{p} 75^{\mathrm{NTR}}$ receptor in the sciatic nerve of rats after neurotmesis (30). Therefore, the increased expression of S-100 in the cMSC and mMSC groups compared to that in the DG and VG groups in our study may be related to a possible differentiation of the transplanted Ad-MSC into Schwann-like cells, as well as to an enhancement of endogenous proliferation of Schwann cells mediated by paracrine effects of Ad-MSC as previously stated.

In the murine neurotmesis model, the functional recovery starts around day 30, with the formation of mature axons and myelin sheaths reaching the threshold at 120 days (25). Therefore, the absence of muscle recovery in the MSC treated groups might be justified by an insufficient time to achieve complete muscle reinnervation (24).

In general, neurotmesis of $10-\mathrm{mm}$ nerve gap produces 
minimal or no functional recovery in rats (3). However, several studies using MSC-based therapy showed positive effects in the sciatic nerve regeneration 6 to 12 weeks after neurotmesis varying from 3-to-10-mm nerve gap $(6,8,23$, $24,26,38-40)$. Our results are in accordance with these findings, indicating that after a neurotmesis of $10 \mathrm{~mm}$, the nerve regeneration is enhanced by MSC transplantation, even in a short period of 35 days.

No morphological differences were observed among the experimental groups related to the inflammatory response in the sciatic nerves treated. However, we can hypothesize that the murine Ad-MSC showed better therapeutic effects on nerve regeneration probably due to their more appropriated response to the murine signaling factors released in the microenvironment after injury.

Although our results show positive effects of the MSC-based therapy in the sciatic nerve regeneration in a murine neurotmesis model, the major limitation of the study is related to the short period of evaluation (35 days) compared to the length of time needed to reach maximum neuroregeneration. Further studies should be performed to confirm the benefits of MSC-based therapy on nerve regeneration, especially canine MSC, aiming at further proving their possible application in clinics in the future.

\section{Conclusions}

Canine and murine Ad-MSC showed positive effects on the sciatic nerve regeneration process in the experimental model of neurotmesis in rats. However, at day 35 the murine MSC showed better functional and electrophysiological results than the canine MSC.

\section{Acknowledgements}

This study was funded by the College of Veterinary Medicine and Animal Science, São Paulo State University "Júlio de Mesquita Filho" (FMVZ-UNESP, campus Botucatu), and the Experimental Research Unit of the School of Medicine of Botucatu (FMB-UNESP). The authors thank the DVM Diego Generoso and Diana Leocata for technical assistance, Juliana Mira for help in statistical analysis and Giovana Boff for contribution to the manuscript correction.

\section{Potential conflict of interest}

The authors have no conflicting financial interest.

\section{References}

1. Ehlers MD. Deconstructing the axon: Wallerian degener- ation and the ubiquitin-proteasome system. Trends Neurosci 2004;27:3-6

2. Millesi H. Factors affecting the outcome of peripheral nerve surgery. Microsurgery 2006;26:295-302

3. Lundborg G. A 25-year perspective of peripheral nerve surgery: evolving neuroscientific concepts and clinical significance. J Hand Surg Am 2000;25:391-414

4. Wang J, Ding F, Gu Y, Liu J, Gu X. Bone marrow mesenchymal stem cells promote cell proliferation and neurotrophic function of Schwann cells in vitro and in vivo. Brain Res 2009;1262:7-15

5. Tyner TR, Parks N, Faria S, Simons M, Stapp B, Curtis B, Sian K, Yamaguchi KT. Effects of collagen nerve guide on neuroma formation and neuropathic pain in a rat model. Am J Surg 2007;193:e1-e6

6. Oliveira JT, Almeida FM, Biancalana A, Baptista AF, Tomaz MA, Melo PA, Martinez AM. Mesenchymal stem cells in a polycaprolactone conduit enhance median-nerve regeneration, prevent decrease of creatine phosphokinase levels in muscle, and improve functional recovery in mice. Neuroscience 2010;170:1295-1303

7. Phinney DG, Prockop DJ. Concise review: mesenchymal stem/multipotent stromal cells: the state of transdifferentiation and modes of tissue repair--current views. Stem Cells 2007;25:2896-2902

8. Luís AL, Rodrigues JM, Geuna S, Amado S, Shirosaki Y, Lee JM, Fregnan F, Lopes MA, Veloso AP, Ferreira AJ, Santos JD, Armada-Da-silva PA, Varejão AS, Maurício AC. Use of PLGA 90:10 scaffolds enriched with in vitro-differentiated neural cells for repairing rat sciatic nerve defects. Tissue Eng Part A 2008;14:979-993

9. Angius D, Wang H, Spinner RJ, Gutierrez-Cotto Y, Yaszemski MJ, Windebank AJ. A systematic review of animal models used to study nerve regeneration in tissue-engineered scaffolds. Biomaterials 2012;33:8034-8039

10. Dezawa M, Takahashi I, Esaki M, Takano M, Sawada H. Sciatic nerve regeneration in rats induced by transplantation of in vitro differentiated bone-marrow stromal cells. Eur J Neurosci 2001;14:1771-1776

11. Keilhoff G, Fansa H. Mesenchymal stem cells for peripheral nerve regeneration--a real hope or just an empty promise?. Exp Neurol 2011;232:110-113

12. Chamberlain G, Fox J, Ashton B, Middleton J. Concise review: mesenchymal stem cells: their phenotype, differentiation capacity, immunological features, and potential for homing. Stem Cells 2007;25:2739-2749

13. Nauta AJ, Fibbe WE. Immunomodulatory properties of mesenchymal stromal cells. Blood 2007;110:3499-3506

14. Caseiro AR, Pereira T, Ivanova G, Luís AL, Maurício AC. Neuromuscular regeneration: perspective on the application of mesenchymal stem cells and their secretion products. Stem Cells Int 2016;2016:9756973

15. Spees JL, Olson SD, Whitney MJ, Prockop DJ. Mitochondrial transfer between cells can rescue aerobic respiration. Proc Natl Acad Sci U S A 2006;103:1283-1288

16. Varejão AS, Cabrita AM, Meek MF, Bulas-Cruz J, Melo- 
Pinto P, Raimondo S, Geuna S, Giacobini-Robecchi MG. Functional and morphological assessment of a standardized rat sciatic nerve crush injury with a non-serrated clamp. J Neurotrauma 2004;21:1652-1670

17. Bertanha M, Moroz A, Almeida R, Alves FC, Acorci Valério MJ, Moura R, Domingues MA, Sobreira ML, Deffune E. Tissue-engineered blood vessel substitute by reconstruction of endothelium using mesenchymal stem cells induced by platelet growth factors. J Vasc Surg 2014;59:1677-1685

18. Okuda K, Kawase T, Momose M, Murata M, Saito Y, Suzuki H, Wolff LF, Yoshie H. Platelet-rich plasma contains high levels of platelet-derived growth factor and transforming growth factor-beta and modulates the proliferation of periodontally related cells in vitro. J Periodontol 2003; 74:849-857

19. Bain JR, Mackinnon SE, Hunter DA. Functional evaluation of complete sciatic, peroneal, and posterior tibial nerve lesions in the rat. Plast Reconstr Surg 1989;83:129-138

20. Dahlhoff M, Emrich D, Wolf E, Schneider MR. Increased activation of the epidermal growth factor receptor in transgenic mice overexpressing epigen causes peripheral neuropathy. Biochim Biophys Acta 2013;1832:2068-2076

21. Carriel VS, Aneiros-Fernandez J, Arias-Santiago S, Garzón IJ, Alaminos M, Campos A. A novel histochemical method for a simultaneous staining of melanin and collagen fibers. J Histochem Cytochem 2011;59:270-277

22. Hood B, Levene HB, Levi AD. Transplantation of autologous Schwann cells for the repair of segmental peripheral nerve defects. Neurosurg Focus 2009;26:E4

23. Orbay H, Uysal AC, Hyakusoku H, Mizuno H. Differentiated and undifferentiated adipose-derived stem cells improve function in rats with peripheral nerve gaps. J Plast Reconstr Aesthet Surg 2012;65:657-664

24. Liu G, Cheng Y, Guo S, Feng Y, Li Q, Jia H, Wang Y, Tong L, Tong X. Transplantation of adipose-derived stem cells for peripheral nerve repair. Int J Mol Med 2011; 28:565-572

25. Tos P, Ronchi G, Papalia I, Sallen V, Legagneux J, Geuna S, Giacobini-Robecchi MG. Chapter 4: Methods and protocols in peripheral nerve regeneration experimental research: part I-experimental models. Int Rev Neurobiol 2009;87:47-79

26. Liu Y, Nie L, Zhao H, Zhang W, Zhang YQ, Wang SS, Cheng L. Conserved dopamine neurotrophic factor-transduced mesenchymal stem cells promote axon regeneration and functional recovery of injured sciatic nerve. PLoS One 2014;9:e110993

27. Hsieh SC, Chang CJ, Cheng WT, Tseng TC, Hsu SH. Effect of an Epineurial-Like Biohybrid Nerve Conduit on Nerve Regeneration. Cell Transplant 2016;25:559-574
28. Dinh P, Hazel A, Palispis W, Suryadevara S, Gupta R. Functional assessment after sciatic nerve injury in a rat model. Microsurgery 2009;29:644-649

29. Cui L, Jiang J, Wei L, Zhou X, Fraser JL, Snider BJ, Yu SP. Transplantation of embryonic stem cells improves nerve repair and functional recovery after severe sciatic nerve axotomy in rats. Stem Cells 2008;26:1356-1365

30. Cartarozzi LP, Spejo AB, Ferreira RS Jr, Barraviera B, Duek E, Carvalho JL, Góes AM, Oliveira AL. Mesenchymal stem cells engrafted in a fibrin scaffold stimulate Schwann cell reactivity and axonal regeneration following sciatic nerve tubulization. Brain Res Bull 2015;112:14-24

31. Boyd JG, Gordon T. Neurotrophic factors and their receptors in axonal regeneration and functional recovery after peripheral nerve injury. Mol Neurobiol 2003;27:277-324

32. Klimaschewski L, Hausott B, Angelov DN. The pros and cons of growth factors and cytokines in peripheral axon regeneration. Int Rev Neurobiol 2013;108:137-171

33. Höke A, Gordon T, Zochodne DW, Sulaiman OA. A decline in glial cell-line-derived neurotrophic factor expression is associated with impaired regeneration after long-term Schwann cell denervation. Exp Neurol 2002; 173:77-85

34. Fu SY, Gordon T. The cellular and molecular basis of peripheral nerve regeneration. Mol Neurobiol 1997;14:67-116

35. Boyd JG, Gordon T. A dose-dependent facilitation and inhibition of peripheral nerve regeneration by brain-derived neurotrophic factor. Eur J Neurosci 2002;15:613-626

36. Salgado AJ, Reis RL, Sousa NJ, Gimble JM. Adipose tissue derived stem cells secretome: soluble factors and their roles in regenerative medicine. Curr Stem Cell Res Ther 2010; 5:103-110

37. Polacek M, Bruun JA, Elvenes J, Figenschau Y, Martinez I. The secretory profiles of cultured human articular chondrocytes and mesenchymal stem cells: implications for autologous cell transplantation strategies. Cell Transplant 2011;20:1381-1393

38. Sun F, Zhou K, Mi WJ, Qiu JH. Repair of facial nerve defects with decellularized artery allografts containing autologous adipose-derived stem cells in a rat model. Neurosci Lett 2011;499:104-108

39. Jia H, Wang Y, Tong XJ, Liu GB, Li Q, Zhang LX, Sun $\mathrm{XH}$. Sciatic nerve repair by acellular nerve xenografts implanted with BMSCs in rats xenograft combined with BMSCs. Synapse 2012;66:256-269

40. Kingham PJ, Kolar MK, Novikova LN, Novikov LN, Wiberg M. Stimulating the neurotrophic and angiogenic properties of human adipose-derived stem cells enhances nerve repair. Stem Cells Dev 2014;23:741-754 